\title{
A new sequenced allelic ladder marker for D1S80 typing
}

Received: 24 October 2003/ Accepted: 11 December 2003 / Published online: 26 February 2004

(C) The Japan Society of Human Genetics and Springer-Verlag 2004

\begin{abstract}
A sequenced allelic ladder marker that contains 32 alleles consisting of alleles 13-44 was developed for D1S80 (MCT118) typing. Each allele, after amplification with D1S80 primers, was cloned to the pT7Blue T-Vector. The plasmid DNAs of these clones were mixed and amplified with D1S80 primers to construct the allelic ladder marker. All the alleles prepared in this study were sequenced in both directions, using two types of forward primers and one reverse primer. The sequencing results revealed that all the alleles in our allelic ladder marker had the correct length corresponding to the allele number. This marker can be used for more effectively typing $D 1 S 80$.
\end{abstract}

Keywords Forensic $\cdot$ DNA typing Variable number of tandem repeat $\cdot D 1 S 80 \cdot$ Allelic ladder marker

The nucleotide sequence data reported are available in the DDBJ database under the accession numbers AB121699-AB121730

K. Fujii $(\bowtie) \cdot$ K. Kasai

Fourth Biological Section,

National Research Institute of Police Science,

6-3-1 Kashiwanoha, Kashiwa,

Chiba 277-0882, Japan

E-mail: fujii@nrips.go.jp

Tel: + 81-4-71358001

Fax: + 81-4-71339153

K. Sekiguchi

Third Biological Section,

National Research Institute of Police Science,

6-3-1 Kashiwanoha, Kashiwa,

Chiba 277-0882, Japan

K. Shimizu

Forensic Science Laboratory,

Miyazaki Prefectural Police Headquarters,

1-8-28 Asahi, Miyazaki 880-8509, Japan

\section{Introduction}

D1S80 (pMCT118, GenBank accession number D28507) is a variable number of tandem repeat locus located on chromosome 1p35-36 with a repeat unit of 16 bp (Nakamura et al. 1988; Kasai et al. 1990). The D1S80 locus is very useful for human identification because of its high polymorphism, especially in the Japanese population (Rand et al. 1992; Sugiyama et al. 1993; TWGFDM 1995). Baechtel et al. (1993) described an allelic ladder marker that consisted of alleles 16-37 and 41 of the locus, but they were not sequenced. Applied Biosystems (Foster City, Calif., USA) supplied the AmpliFLP D1S80 Allelic Ladder consisting of alleles 14 and $16-41$ of the locus, but they only sequenced alleles 14, 16, 18, and 24 in the allelic ladder marker. In addition, Applied Biosystems stopped supplying the AmpliFLP D1S80 Allelic Ladder in 2003. When using the AmpliFLP D1S80 Allelic Ladder, we were not able to type alleles larger than allele 41. However, the alleles larger than allele 41 have been observed with a frequency of about $1 \%$ in the Japanese population (TWGFDM 1995). In order to type them, an allelic ladder marker that contains some alleles larger than the allele 41 is required. Here, we constructed a new allelic ladder marker consisting of alleles 13-44 of the DIS80 locus and confirmed that all the alleles had the correct length corresponding to the allele number by sequencing.

\section{Materials and methods}

Blood samples were collected from 23 Japanese individuals that were heterozygous at the D1S80 locus. Appropriate informed consent was obtained from each individual. DNA samples were purified from their blood samples using a QIAamp DNA Mini Kit (Qiagen, Hilden, Germany). DNA samples were amplified with D1S80 primers (Kasai et al. 1990), and each allele was separated on a $2 \%$ agarose gel or by denaturing high-performance liquid chromatography using a WAVE system (Transgenomic, Omaha, Neb., USA). Each separated D1S80 allele was 


\section{a}

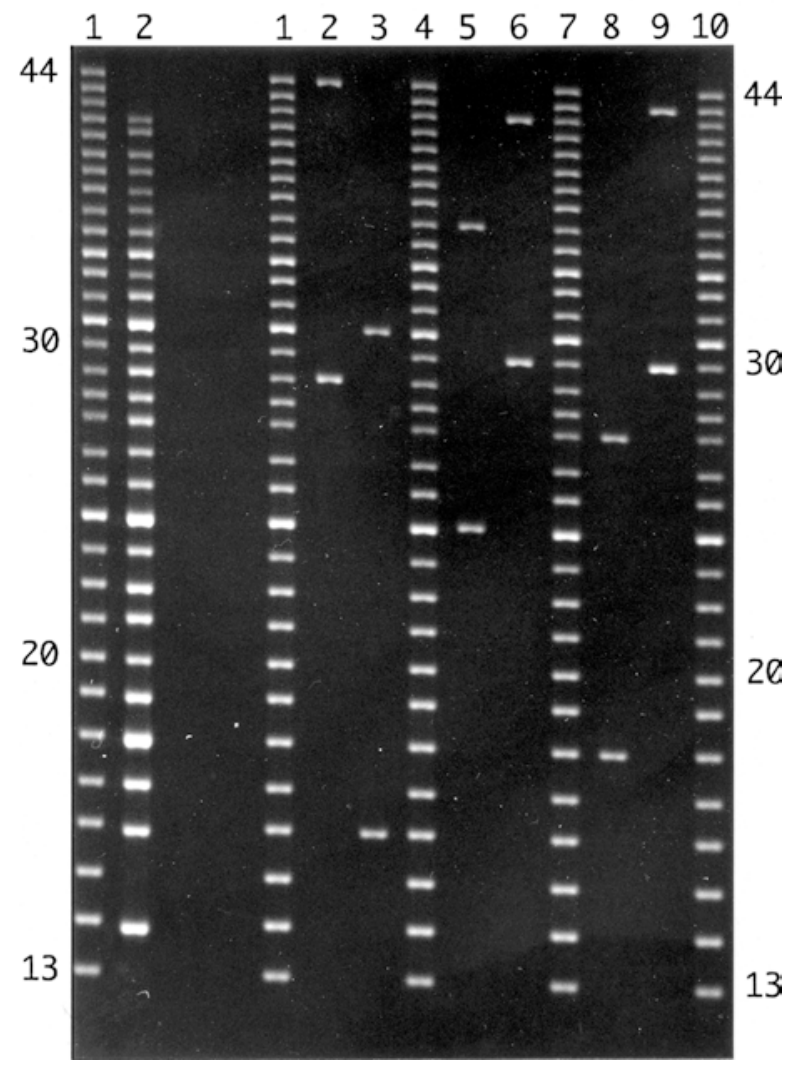

Fig. 1a, b A new sequenced allelic ladder marker for D1S80 typing. a Lane $1 \mathrm{~A}$ new allelic ladder marker consisting of alleles 13-44, lane 2 AmpliFLP D1S80 Allelic Ladder (Applied Biosystems) consisting of alleles 14 and 16-41. b Lanes 1, 4, 7 , 10 A new allelic ladder marker, lanes 2, 3, 5, 6, 8, 9 PCR products of blood samples amplified with D1S80 primers (lanes 2, 29-44; lanes 3, 16-31; lanes 5, 24-36; lanes 6, 30-42; lanes 8, 18-27; lanes 9, 30-43)

cloned to the pT7Blue T-Vector (Novagen, Madison, Wis., USA).

The plasmid DNAs containing the alleles 13-44 of the D1S80 locus were mixed and amplified with D1S80 primers to construct an allelic ladder marker. The PCR was performed using 0.14-1.2 fg of each plasmid DNA in a $100-\mu$ reaction volume with a TaKaRa PCR Thermal Cycler Dice (Takara Bio, Otsu, Japan). The PCR reaction mixture contained $1 \times$ Ex Taq buffer $\left(2 \mathrm{mM} \mathrm{Mg}^{2+}\right.$ Plus, Takara Bio), $0.2 \mathrm{mM}$ dNTPs, $0.05 \mathrm{U} / \mu \mathrm{l}$ TaKaRa Ex Taq HS (Takara Bio), and $1.4 \mu \mathrm{M}$ of each of the D1S80 primers. The thermal cycle was performed at $95^{\circ} \mathrm{C}$ for $1 \mathrm{~min}$, followed by 27 cycles at $94^{\circ} \mathrm{C}$ for $1 \mathrm{~min}, 65^{\circ} \mathrm{C}$ for $1 \mathrm{~min}$, and $70^{\circ} \mathrm{C}$ for $1 \mathrm{~min}$. A final extension was performed at $70^{\circ} \mathrm{C}$ for $10 \mathrm{~min}$.
All the plasmids containing the alleles 13-44 were sequenced. Four hundred nanograms of the plasmid DNA was sequenced with the T7 promoter primer, with the M13-21 primer, and with the Forward 2 primer (5'-CAGCC CAAGG AAGAC AGACC-3') reported by Watanabe and Shimizu (2002). The sequence reaction with the $\mathrm{T} 7$ promoter primer was performed at $95^{\circ} \mathrm{C}$ for $5 \mathrm{~min}$, followed by 25 cycles at $95^{\circ} \mathrm{C}$ for $30 \mathrm{~s}, 46^{\circ} \mathrm{C}$ for $10 \mathrm{~s}$, and $60^{\circ} \mathrm{C}$ for $4 \mathrm{~min}$. The sequence reaction with $\mathrm{M} 13-21$ was performed at $95^{\circ} \mathrm{C}$ for $5 \mathrm{~min}$, followed by 25 cycles at $95^{\circ} \mathrm{C}$ for $30 \mathrm{~s}$, and $60^{\circ} \mathrm{C}$ for $4 \mathrm{~min}$. The sequence reaction mixture of the T7 promoter primer and of the M13-21 primer contained 5\% dimethyl sulfoxide and $5 \%$ glycerol. The sequencing reaction with the Forward 2 primer was performed with the method described by Watanabe and Shimizu (2002).

\section{Results and discussion}

An allelic ladder marker consisting of the alleles 13-44 for use in D1S80 typing was constructed (Fig. 1). The forward sequences were obtained from all the plasmids with the $\mathrm{T} 7$ promoter primer and the Forward 2 primer, and the reverse sequences were obtained with the M13-21 primer. From the sequence data of the alleles 13-44, 13 types of repeat units were obtained, and the repeat units have been reported previously (Fig. 2, Duncan et al. 1997; Arakura et al. 1998; Liu et al. 1999; Watanabe and Shimizu 2002). The repeat units 2-13 consisted of $16 \mathrm{bp}$, and their sequences were designated as RMRRA CCACH RGVAA G. The first four repeats and the last three repeats were conserved expect for the allele 21 . The allele 21 appears to contain a nucleotide substitution in the 19th repeat. However, we do not know whether the nucleotide substitution occurred in the PCR amplification before the cloning or was derived from an individual who provided the allele. All the alleles used in this study contained the same $5^{\prime}$ and $3^{\prime}$ franking region sequences that were similar to the sequence reported by Kasai et al. (1990). The lengths of all the alleles in this study were calculated using the following formula reported by Sekiguchi et al. (1993): $116 \mathrm{bp}\left(5^{\prime}\right.$ franking region) $+14 \mathrm{bp}$ (first repeat) $+16(n-1) \mathrm{bp}$ (second repeat through last repeat $)+32 \mathrm{bp}\left(3^{\prime}\right.$ franking region $)=146+16 n \mathrm{bp}$, where $n$ is the allele number. The sequencing result revealed that all the alleles in our allelic ladder marker had the correct length corresponding to the allele number, and we think that this marker can be used for more effectively typing D1S80. Takara Bio now supplies the TaKaRa D1S80 Allelic Ladder using the method decribed in this study. 


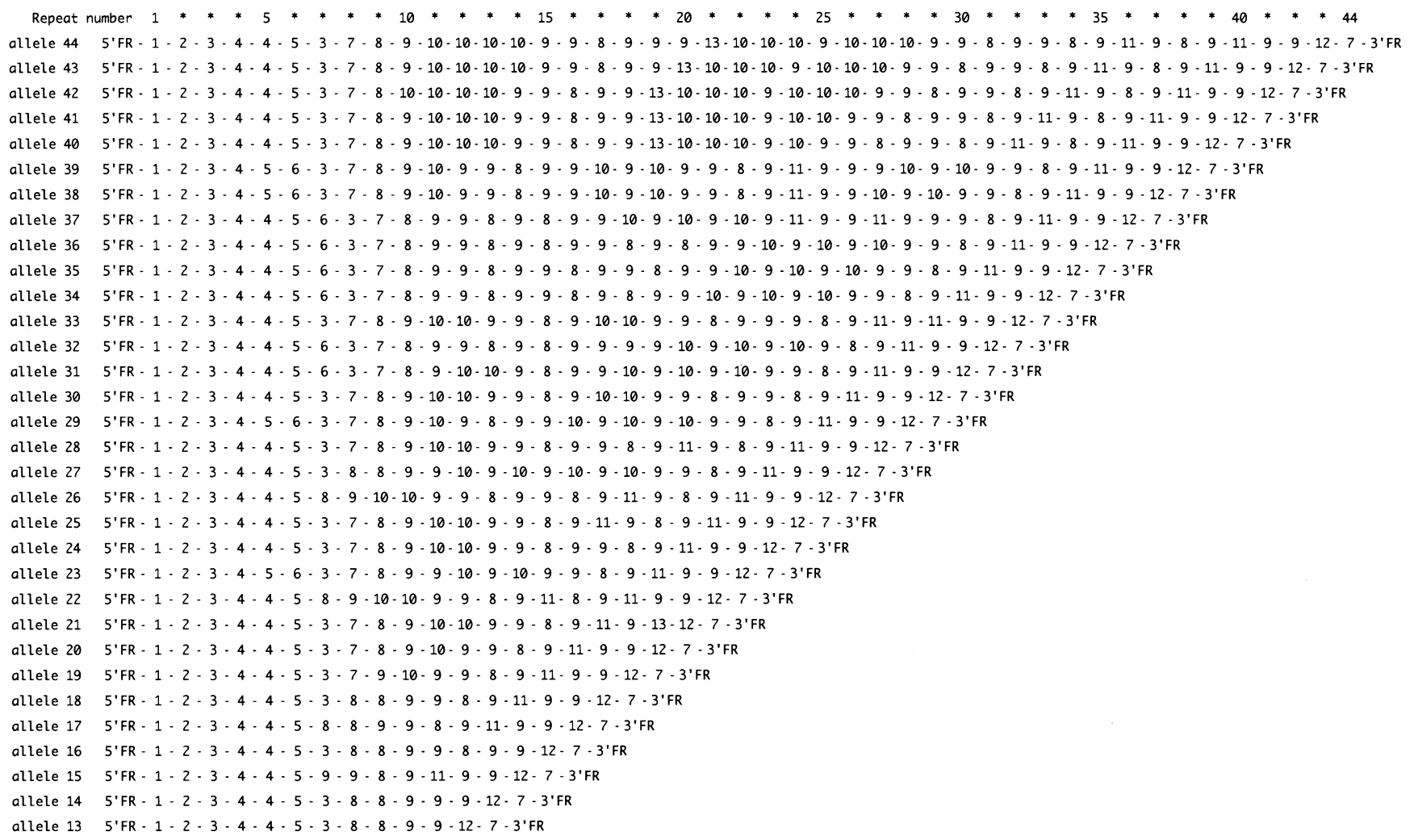

The sequences of the repeat units (1-13) and the 5' and 3' franking regions. 1:TCAGC CCAAG GAAG 2:ACAGA CCACA GGCAA G 3:GAGGA CCACC GGAAA G 6: GAGGA CCACA GGCAA G 7:GAAGA CCACC GGCAA G 8:GAGGA CCACC GGCAA G

\section{4: GAAGA CCACC GGAAA G 5: GAAGA CCACA GGCAA G} 9: GAGGA CCACC AGGAA G 10: GAGGA CCACC AGCAA G

11: GAGAA CCACC AGGAA G 12: GAGGA CCACT GGCAA G 13: GAGGA CCACC GGGAA G

5'FR:GAAAC TGGCC TCCAA ACACT GCCCG CCGTC CACGG CCGGC CGGTC CTGCG TGTGA ATGAC TCAGG AGCGT ATTCC CCACG CGCCA GCACT GCATT CAGAT AAGCG CTGGC TCAGT G

3'FR: CCTGC AAGGG GCACG TGCAT CTCCA ACAAG AC

Fig. 2 Sequence structures of alleles $13-44$ of the D1S80 locus

\section{References}

Arakura A, Liu C, Ota M, Fukushima H (1998) Subtyping and characterization of D1S80 alleles in a Japanese population using PCR-RFLP. Int J Legal Med 111:183-187

Baechtel FS, Smerick JB, Presley KW, Budowle B (1993) Multigenerational amplification of a reference ladder for alleles at locus D1S80. J Forensic Sci 38:1176-1182

Duncan GT, Balamurugan K, Budowle B, Smerick J, Tracy ML (1997) Microvariation at the human D1S80 locus. Int J Legal Med 110:150-154

Kasai K, Nakamura Y, White R (1990) Amplification of a variable number of tandem repeats (VNTR) locus ( $p M C T 118$ ) by the polymerase chain reaction (PCR) and its application to forensic science. J Forensic Sci 35:1196-1200

Liu C, Arakura A, Takayanagi K, Asamura H, Ota M, Fukushima H (1999) DIS80 subtyping by PCR-RFLP: new nomenclature and further characterization. Leg Med 1:210-216
Nakamura Y, Carlson M, Krapcho K, White R (1988) Isolation and mapping of a polymorphic DNA sequence ( $p M C T 118)$ on chromosome 1p [D1S80]. Nucl Acids Res 16:9364

Rand S, Puers C, Skowasch K, Wiegand P, Budowle B, Brinkmann B (1992) Population genetics and forensic efficiency data of 4 AMPFLPs. Int J Legal Med 104:329-333

Sekiguchi K, Sakai I, Mizuno N, Yoshida K, Kasai K, Sato H, Seta S (1993) DNA sequence analysis of PCR-amplified products of MCT118 locus in Japanese DNA samples (in Japanese). Rep Nat Res Inst Police Sci 46:172-176

Sugiyama E, Honda K, Katsuyama Y, Uchiyama S, Tsuchikane A, Ota M, Fukushima H (1993) Allele frequency distribution of the D1S80 ( $p M C T 118$ ) locus polymorphism in the Japanese population by the polymerase chain reaction. Int $\mathrm{J}$ Legal Med 106:111-114

TWGFDM (Technical working group for forensic DNA typing method) (1995) Reassessment on frequency distribution of MCT118 and HLADQ $\alpha$ alleles and genotypes in Japanese population along with the comparison with other population data (in Japanese). Rep Nat Res Inst Police Sci 48:171-185

Watanabe G, Shimizu K (2002) DNA sequence analysis of long PCR amplified products at the D1S80 locus. Leg Med 4:37-39 